\title{
The Sudan Peritoneal Dialysis Program: Three Years of Momentum
}

\author{
Hasan Abu-Aisha ${ }^{\mathrm{a}}$, Elwaleed AM Elhassan ${ }^{\mathrm{b} *}$, Sarra Elamin ${ }^{\mathrm{c}}$ \\ a. FRCP, Professor of medicine and nephrology, National Ribat University, Khartoum, Sudan \\ b. MD, Division of Renal Diseases and Hypertension, University of Colorado Health Sciences Center, Aurora, USA \\ c. MRCP, Sudan Peritoneal Dialysis Program
}

\begin{abstract}
Introduction: End stage renal disease (ESRD) is a significant social and economic burden on Africa. Continuous ambulatory peritoneal dialysis (CAPD) was introduced as a national service in the Sudan three years ago. An overview of the Sudan Peritoneal Dialysis Program is presented.
\end{abstract}

Review: As a national scheme, the Sudan PD program was sequentially launched in seven centers in Sudan. CAPD was the modality exclusively utilized. The total number of patients enrolled by July 2008 was 232 . Patients' mean age was 43 years, $23.5 \%$ were children, $63 \%$ were males, and $19 \%$ were diabetic.

PD was the initial dialysis modality for $54.7 \%$ of patients, $43.5 \%$ were referred from HD, and $1.9 \%$ had a failed kidney transplant. The majority of patients (62.3\%) chose PD as the preferred dialysis modality, while the remainder were referred from $\mathrm{HD}$ to $\mathrm{PD}$ because of vascular access problems or hemodynamic instability. One third of patients required assisted PD, and 17\% needed urgent initiation of dialysis.

The cumulative peritonitis rate was one episode per 10.8 patient months. Touch contamination, urgent initiation of dialysis and execution of dialysis by family members other than trained helpers were among the identified predisposing factors.

Conclusion: The establishment and operation of the Sudan PD program over the past three years have indicated that it is a promising project with multifaceted success. There is, however, an ongoing need for retraining of patients and staff. Early selection and careful preparation for potential PD candidates is also of vital importance to reduce the need for urgent dialysis initiation.

* Corresponding author; Division of Renal Diseases and Hypertension, University of Colorado Health Sciences Center, P.O.Box 6508, Aurora, CO 80045, USA.

E mail: Elwaleed.Elhassan@UCHSC.edu
Key words: peritoneal dialysis, CAPD, renal replacement therapy, Sudan, Africa

\section{Introduction}

End stage renal disease (ESRD) is a substantial social and economic problem worldwide, and one that is increasing. Both incidence and prevalence of treated ESRD are increasing [1]. The global total cumulative cost for renal replacement therapy (RRT) is over one trillion US\$. The cost and complexity of RRT make it extremely challenging for developing countries.

Considerable progress has been made during the past decade in the practice and theory of peritoneal dialysis (PD) for ESRD [2]. First, the widespread introduction of automated peritoneal dialysis (APD), whereby dialysis fluid is exchanged frequently while the patient is asleep, improves ultrafiltration and further reduces the time that the patient needs to devote to dialysis procedures. Second, the introduction of icodextrin dialysis fluid, containing a polyglucose that maintains a long-term osmotic pressure gradient, has reduced the problem of negative ultrafiltration during the night exchange or long day exchanges [3]. Third, the improvements in connector technology and training techniques have markedly reduced peritonitis frequency. The introduction of biocompatible peritoneal dialysis fluids has reduced dialysis discomfort and promises further reduction in peritonitis frequency and better long-term preservation of peritoneal function.

Recent studies have shown an adjusted survival advantage for PD compared with hemodialysis (HD), particularly during the first 2 years of dialysis $[4,5]$. Technique failure rates for $\mathrm{PD}$ at $7 \%$ to $11 \%$ per year [6] are generally higher than for HD, either due to patient "burn-out" and reduced motivation due to increasing morbidity, recurrent peritonitis, or peritoneal membrane failure. The "integrated care" approach to ESRD [7] does not consider this relatively higher failure rate to be a contraindication. This concept advocates that HD, $\mathrm{PD}$, and renal transplantation should all be offered to the 
patient in an unbiased way, and that all three modalities may be a part of treatment during the patient's lifetime. For many, PD will be a reasonable initial choice, hopefully followed by renal transplantation, but otherwise with timely conversion to HD should complications arise from PD. $[8,9]$

PD is an efficient dialysis modality that can be tailored to the individual patient's clinical and social requirements. It permits a high degree of social rehabilitation and travel freedom. Given an informed choice, approximately half of patients will choose PD [10]. However, about $20 \%$ of patients will be unsuitable for PD either due to abdominal problems (e.g. hernias, multiple operations), physical disabilities (e.g. hemiparesis), or psychological problems (e.g. dementia, noncompliance). Thus, the overall rates of newly initiated PD cannot be expected to be as high.

PD remains underutilized in many countries. Blake and Finkelstein speculated on the reasons for the decline in chronic PD utilization in the United States and Canada [11]. They suggested that a variety of factors contributed to this decline, such as the increased age of patients, the increased severity of illness, the increased availability of HD facilities, the challenges of running a PD unit with a limited number of patients, a lack of focus on the education of the patient with chronic kidney disease (CKD), and the inadequate training of nephrologists in chronic PD therapy.

Regarding Africa, scant published data exits about the prevalent use of chronic PD in the continent. A recent survey published in the previous issue of this Journal provided important information about the distribution of RRT in different African countries [12]. It has shown that PD is routinely available in 12 countries ( 5 in North Africa, 7 in South Africa), and that it is only being delivered in the public sector. In most of these countries it has been introduced recently and most units offer acute hard catheter PD, only a few units offer continuous ambulatory peritoneal dialysis (CAPD). The total number of PD patients is around 1,800 and the prevalence is less than $15 \mathrm{pmp}$ in all countries, while it is more than 100 pmp in Western Europe. It is often more expensive than HD because all components, including fluids are imported.

Sudan is the largest country in Africa with a total area of 2,505,810 sq km, and a total population of about 36 million. The annual population growth rate is $2.2 \%$ and the rural population constitutes about $60 \%$. The Median age of the population is 20.4 years while the percentage of those 60 years of age and above is $5.8 \%$.

There is no national renal registry in the Sudan but we estimate the prevalence of CKD to be about $5.1 \%$ among the adult population, based on a pilot study on the prevalence of CKD in police forces households in Khartoum, Sudan (unpublished data). ESRD is projected to affect 5400 new cases per year, with an estimated incidence of 150 new cases per million of population per year. Center-based HD is limited and restricted to few major towns. Trained staff and dialysis machines are scarce. Although the HD treatment cost is mainly borne by the federal government, it is far from enough to address the growing numbers of ESRD patients. Kidney transplantation is offered in few major hospitals. The growing number of successful transplants is encouraging. However, many patients are hampered from that option by the prohibitive cost of preparatory investigations and life-long medications as well as some severely advanced comorbidities. Hard-catheter acute PD is widely available as a temporary life-saving intervention for uremic patients who tend to present late.

As of December 2007, the total number of patients on regular hemodialysis in the Sudan was 2750 . The registered number of HD centers in the country is 27 . The total number of qualified nephrologists is 20. One third of all patients on HD live outside the capital Khartoum; most of them are being looked after by non-nephrologists. The dialysis treatment cost is borne by the Federal government and the cost of a single HD session is about 100 US\$. Most patients undergo twice weekly HD that is being delivered in 21 public and 6 private centers. This brings the total annual cost to 10,400 US $\$$ per patient. The current annual PD cost for a single patient is 11,680 US\$ and is all delivered at public hospitals. The total physician and facility reimbursement for HD is 70 US\$ per session while none has been established for PD thus far. The transplantation program has recently been rejuvenated. The number of renal transplant operations has steadily increased with a total of 430 operations performed in the last seven years, all from living donors [13].

\section{Organization of the Sudan PD Program}

CAPD was introduced as a national service for the first time in Sudan in June of 2005. The Sudan PD program is fully funded by the Federal State and the CAPD fluids are provided by the Central Medical Supplies (CMS) Corporation. As a national experiment, the program was launched in five adult and two pediatric centers in Greater Khartoum, the capital city of the Country. By the middle of 2008 the first regional center outside Khartoum was established in Medani, the capital of the Gezira State. In each center there are 1-2 nephrologists or experienced general physicians and trained nurses (Table 1).

The Sudan PD Program has a central headquarters; it utilizes an integrated system for all patients with unified 
Table 1: Distribution of PD patients between different PD centers by the end of July 2008

\begin{tabular}{lll}
\hline \multirow{2}{*}{ Center } & \multicolumn{2}{l}{ Total patients enrolled } \\
\cline { 3 - 3 } & Frequency & Percentage (\%) \\
\hline Ribat University Hospital & 101 & 43.5 \\
Military Hospital & 40 & 17.2 \\
Soba Pediatric Center & 40 & 17.2 \\
Khartoum Teaching Hospital & 19 & 8.2 \\
Jaafar Ibn Children's Hospital & 14 & 6 \\
Ibn Sina Specialized Hospital & 10 & 4.3 \\
Medani Center for Kidney Disease and Surgery & 8 & 3.4 \\
Total & 232 & 100 \\
\hline
\end{tabular}

databases, protocols, and fluid transfer arrangements. Monthly meetings are carried out to discuss all individual patient problems, update center reports and to design protocols for preoperative preparation, follow up, peritonitis and exit site infection treatments. Home visits are carried out and continuing staff education programs are regularly provided. In subsequent years, the program will accommodate more centers after careful selection. The most important criteria for center inclusion are "an enthusiastic nephrologist interested in PD and willing to work in a team fashion" and a "willing hospital administration". Arrangements to meet other cost items are still being discussed with CMS.

The "integrated multi-center" approach is probably the most important aspect of the program organization. The program came to being thanks to the National Ribat University (NRU) which accepted the program as a research project by the Principal Investigator (HA). NRU supported the infrastructure needed to accommodate the "Leading Center" in the university premises. The CMS Corporation, which is a Federal State organization dealing with medical supplies to the whole Country, agreed to "give the proposed integrated multi-center program a try" as a free medical service to some ESRD patients selected by the organizers of the program. Credit must also be given to all the team members of the seven centers, who fully cooperated and enthusiastically accepted the unified protocols and regularly attended the monthly meetings which are held alternately in participating centers.

\section{Patients' characteristics}

Between June 2005 and July 2008, 232 patients joined the Sudan PD program. Patients' mean age was 43 years, with a range of 9 months to 81 years, $23.5 \%$ of all patients belonged to the pediatric age group, $63 \%$ of patients were males, and $19 \%$ were diabetic.

A little over half of the patients resided outside Khartoum. Some of them come from as far as Port Sudan, about a thousand kilometer North East of Khartoum, and Nyala, close to the Western border of the country, about a thousand kilometer West of Khartoum. CAPD was considered the only option for a lot of them because of unavailability of HD facilities in those regions of the country (Figure 1).

PD was the initial dialysis modality for $54.7 \%$ of patients, $43.5 \%$ were referred from $\mathrm{HD}$, and $1.9 \%$ had a failed kidney transplant. The majority of patients chose PD $(62.3 \%)$ as their preferred dialysis modality, while $8 \%$ of patients were referred to PD because they were not medically suitable for HD, and $29.7 \%$ were referred to PD because of vascular access problems. Almost one third of patients required assisted PD, and about $17 \%$ of patients required urgent initiation of dialysis.

\section{Peritonitis Rates}

The cumulative peritonitis rate at the end of the first year was one episode per 21.5 patient month or 0.55 episodes per year. This was well within the acceptable range recommended by the ISPD guidelines; one episode per 18 patient-months or 0.67 episode per year at risk [14], though some individual center did not quite attain the targeted range. By July of 2008, however, the overall rate had dropped to one episode per 10.8 patient months. This deterioration in efficacy was probably due to staff instability, as nurses were not always fully assigned to the program and many of them were transferred by prospective hospital administrations to other departments at times of seeming crisis. This shortcoming has largely 
Figure 1: Sudan map showing the distribution of PD patients (numbers) between different federal states

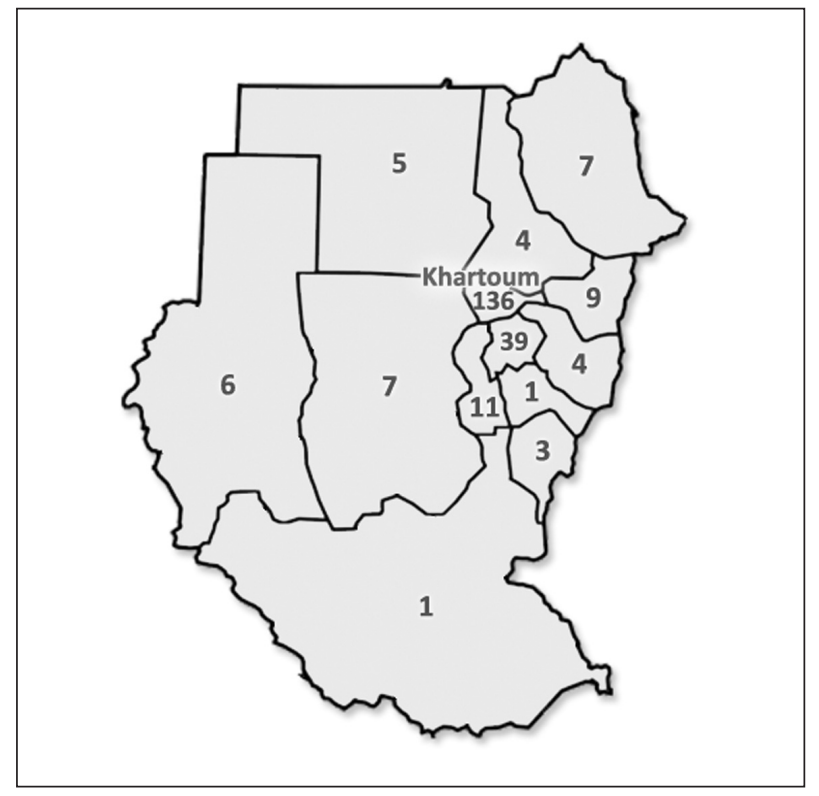

been overcome now and the latest (November 2008) peritonitis rates were recorded as one episode per 11.3 patient months. We hope this trend towards improvement will continue over the coming months.

Touch contamination was incriminated in a considerable proportion of peritonitis episodes. Touch contamination was defined as a break in the sterile technique by which dialysis is supposed to be performed. Examples encountered were failure to wash or dry hands appropriately before performing the procedure and inattention to avoid the connector tips contamination when connecting and disconnecting the transfer set and the twin bag. Problems seen with children included execution of dialysis by family members other than trained helpers and, in general, less meticulous hygienic adherence. For logistical reasons, young children are trained to utilize appropriate portions of the same 2-liter adult bags for CAPD and discard the remainder. A concern is that some parents might be re-using the bags for "finding it difficult to throw those precious bags away." Urgent dialysis was also recognized as an important predictor of peritonitis.

The culture-negative peritonitis rate was 53\%; higher than the maximum of $20 \%$ allowed by the ISPD recommendations [14]. There are several difficulties which impede implementing the appropriate processing technique of dialysate samples on patient suspected of having peritonitis as advised by the ISPD guidelines in our setting. Large volume centrifuging devises are not available. Blood culture bottles are expensive and not uniformly available. The culture procedure done in most of our laboratories is injecting a few milliliters of the dialysate directly into solid culture media and checking daily for growth. Storage of the rest of whole bags in an incubator is performed in some centers in order to encourage growth while re-testing the fluid daily.

The cumulative peritonitis rate stresses the ongoing need for retraining of the patients and the program staff. Since the system is an integrated system, the difficulties met by each center are discussed in detail and team-members give advice freely. Therefore, it is very likely that mistakes will be corrected and records improved. The appreciation -incentive system has also proven very effective: the "Best Center" in patient care, "Least Peritonitis," "Best Research Paper" etc, are looked upon as achievements that are worth competing for.

Proper hand hygiene techniques need to be well-taught and persistently respected by patients and medical staff. Early selection and careful preparation for potential PD candidates is also of vital importance to obviate the need for urgent dialysis initiation.

\section{Conclusion}

The Sudan Peritoneal Dialysis Program is now a reality and is promising to serve a large sector of ESRD patients in the Sudan. The total cost of therapy is looked upon as cost-effective as it excludes hospital admission charges and working days loss. The treatment is provided free of charge. However, a major current problem is the cost of laboratory investigations and medications. Ways and means are being sorted out to help patients with these in order to improve the performance of the procedure and assure its continued success.

Challenges also include maintaining a satisfactorily low infection rate which does require continuous training and retraining as well as meticulousness in part of the patient and providers. Overall, PD is an important addition to RRT armamentarium in the Sudan. It probably should be considered as the treatment of first choice for most ESRD patients as advocated in the integrated dialysis care strategy.

\section{References}

1. Locatelli F, Valderrabano F, Hoenich N, Bommer J, Leunissen K, Cambi V. Progress in dialysis technology: membrane selection and patient outcome. Nephrol Dial Transplant. 2000 Aug;15(8):1133-9.

2. Heaf J. Underutilization of Peritoneal Dialysis. JAMA 2004 Feb 11;291(6):740-2. 
3. Wolfson M, Ogrinc F, Mujais S. Review of clinical trial experience with icodextrin. Kidney Int Suppl. 2002 Oct;(81):S46-S52.

4. Heaf JG, Løkkegaard H, Madsen M. Intitial survival advantage of peritoneal dialysis over hemodialysis. Nephrol Dial Transplant. 2002 Jan;17(1):112-7.

5. Korevaar JC, Feith GW, Dekker FW, van Manen JG, Boeschoten EW, Bossuyt PM, Krediet RT; NECOSAD Study Group. Effect of starting with hemodialysis compared to Peritoneal dialysis in patients new on dialysis treatment: a randomized controlled trial. Kidney Int. 2003 Dec;64(6):2222-8.

6. Kawaguchi Y. Peritoneal dialysis as a long-term treatment: comparison of technique survival between Asian and Western populations. Perit Dial Int. 1999;19 Suppl 2:S327-S328.

7. Davies SJ, Van Biesen W, Nicholas J, Lameire N. Integrated care. Perit Dial Int. 2001;21 Suppl 3:S269-74.

8. Blake PG. Integrated end-stage renal disease care: the role of peritoneal dialysis. Nephrology Dial Transplant. 2001;16 Suppl 5:61-6.
9. Mendelssohn DC, Pierratos A. Reformulating the integrated care concept for the new millennium. Perit Dial Int. 2002 Jan-Feb;22(1): 5-8.

10. Golper T. Patient education: can it maximize the success of therapy? Nephrol Dial Transplant. 2001;16 Suppl 7:20-4.

11. Blake PG, Finkelstein FO. Why is the proportion of patients doing peritoneal dialysis declining in North America? Perit Dial Int. 2001 Mar-Apr;21(2):107-14.

12. Aziz Almatri, Elwaleed Elhassan, Hasan Abu-Aisha. AJNT. 2008 Sep;1(1):9-14

13. Wafa Ali Obeid. Review of Sudan Renal Replacement Program; Past, Present and Future: An official publication from the National Center for Kidney Diseases and Surgery. Kharotum: Wadaha Printing Press. 2007. p 6-13.

14. Piraino, B, Bailie, GR, Bernardini, J, Boeschoten E, Gupta A, Holmes C, Kuijper EJ, Li PK, Lye WC, Mujais S, Paterson DL, Fontan MP, Ramos A, Schaefer F, Uttley L; ISPD Ad Hoc Advisory Committee. Peritoneal dialysisrelated infections recommendations: 2005 update. Perit Dial Int. 2005 Mar-Apr;25(2):107-31. 